\title{
Robotic Art and Cultural Imagination
}

\author{
Bojana Romic \\ School of Arts and Communication \\ Nordenskiöldsgatan 1, 21119 Malmö, Sweden \\ bojana.romic@mau.se
}

\begin{abstract}
In this article, I aim to accentuate the importance of the cultural imagination about robots, observing it 'as a mixed register of fantasy and an actual practice' (Kakoudaki 2007, 165). I emphasise the field of robotic art, which, I argue, is in a fluid state of exchange with other areas of robotic research, equally benefiting from the larger context of the cultural imagination about robots.
\end{abstract}

Furthermore, I discuss artworks that offer a valuable commentary on robots even though they are not defined as robotic art in a narrow sense (Penny 2013), given that they feature only the representation of robots or robot-like characters. Nevertheless, these artworks contribute to the circulation of symbolic registers that revolve around the multifaceted figure of a robot.

Robot. Robotic Art. Cultural imagination. Cultural robotics.

\section{INTRODUCTION}

In recent years, there has been an ongoing debate about the notion and (possible) function of robotic culture. With the development of the new generation of drones, as well as the advancement in social and health robotics, questions about the robot culture seem to open a variety of discussions (Kim \& Kim 2013, Samani et al. 2013, Šabanović et al. 2014, Koh et al. 2015). Such discourses begin with the inquiry on how we can grasp the notion of culture ${ }^{1}$ as an umbrella term for the range of habitual and technological practices, in relation to the equally heterogeneous field of robotics. The term robot covers a broad range of meanings that communicate with the common cultural imagination about robots fed by the literature and popular culture. Typically, a robot is considered a mechanical entity that possesses a certain level of autonomy, agency and choice (Kakoudaki 2007).

I do not delve into detail regarding different aspects of scientific research (e.g. HRI, computer vision, social robotics, artificial intelligence (Al), locomotive qualities, etc.) but observe it as a whole.

Whilst I acknowledge the importance of genderrelated questions in cultural robotics (Eyssel 2012, Vlachos, Jochum and Schärfe 2016), they are not the focus of my article. I take into account that depending on the circumstances and/or artistic intention, a robot may be female, male or neither. Furthermore, I observe 'culturally odourless' (Borggreen 2011, 42) aspects of cultural imagination: I do not probe deeper into ideological, religious and societal reasons for imaginaries that are characteristic of particular societies (e.g. Japanese, US, Korean, etc.). Instead, I focus on phenomenological aspects of cultural imagination in robotic art in general.

\section{WHAT IS A ROBOT CULTURE?}

Although the study of culture has a long history in the humanities and the social sciences (Šabanović et al. 2004), only recently has culture become an area of study in the field of robotics:

\footnotetext{
The emerging discipline of cultural robotics [...] defines culture as a notion which is not only attributed to humans, but also encompasses the cultural exchanges between robots, robots and humans, as well as other intellectual and emotional entities (Samani et al. 2013, 2).
}

This definition includes animals as well, given that they are social and emotional beings and may have strong attachments to their groups or packs. There are interesting examples of research that has closely examined the experimental prototypes of a mixed community and has tried to determine the impact of robots on the ecosystem ${ }^{2}$. These experiments show the alteration of animal behaviour when robots are introduced to the closed circuit.

In Western societies, we are witnessing the introduction of animal-like social or personal robots, such as Paro (therapeutic robot, Intelligent System 
Co.), Probo (intelligent, autonomous robot that interacts with hospitalised children, Free University Brussels), iCat (social robot for children and adults, Philips), Huggable (robotic companion for healthcare, education and social communication, Massachusetts Institute of Technology Media Lab) and many others. Such robotic companions are expected to be easily accepted by people, firstly due to the robots' pet-like qualities and the already established relationships between people and animals (Coeckelbergh 2011); secondly, people are not challenged by the 'uncanny valley'3 in terms of robots' appearance (Mori 1970). Besides pet-like robots, other technological agents operate alongside humans as health workers, educators, playmates or even intimate partners (Turkle 2011).

On the other hand, relevant robotic studies have been conducted outside the centres and the laboratories that have pursued research for instrumental purposes, specifically in the area of art and cultural practices (Penny 2013), as well as in doit-yourself (DIY) robotics. Such research offers a broader space for dialogue and rethinking humanrobot interaction (HRI) approaches, iconic stereotypes and ethical concerns. It can even be argued that robotic art and culture has an additional quality of a 'testing space' for an outspread cultural imagination about robots. An example is Rodney A. Brook's six-legged insect robot Genghis (1988), which he describes as 'an artificial agent with a "wasplike" personality' that 'chased and scrambled according to its will' (Dixon 2004, 31). Currently, we can find a whole range of both commercial insectlike robots (e.g. Robugtix T8 spider, Amoeba Robotics Ltd.; Hexapod collection for hobbyists, Lynxmotion) and DIY spider robots.

\section{As Penny notes:}

Cultural robotics is a highly charged interdisciplinary test environment in which the theory and the pragmatics of technical research confronts the phenomenological realities of physical and social being in the world, and the performative and processual practices of the arts $(2013,147)$.

Similarly, Šabanović, Bennett and Lee $(2014,2)$ suggest:

\begin{abstract}
Robotics as a culture-aware practice should incorporate an understanding of scientific practice as a cultural phenomenon and include the critical study of cultural meanings and values as they are defined outside of robotics labs into the development of new robotic technologies.
\end{abstract}

For this reason, the authors propose the phrase culturally robust robotics, which takes culture into account as a broad context in which robot design and use occur. This can be interpreted as an attempt to bridge the persisting gap between the actual robotic technological research and the cultural imagination about robots fuelled by popular initiatives, especially in film, literature and visual and sonic arts.

\section{ROBOTS: IN BETWEEN REAL AND IMAGINARY}

In recent decades, cinema - as a popular medium capable of reaching broad audiences worldwide has produced many fictional robots that have gained a considerable fan base, especially since the advent of the Internet (Jenkins 2006a). These fictional robots that appear on film and other popular media greatly influence people's ideas about them. As noted by Kakoudaki, 'the robot is a figure of fiction and science fiction which, despite its un-reality channels feelings about culture and technology, difference and justice, often in indirect ways' (2007, 165). Kakoudaki carefully avoids the binary opposition between 'real' (produced) and phantasmal robots, arguing that the two approaches fuel each other - we can observe the presence of a robot as a cultural figure in popular media for almost a century now.

Likewise, we can find analogues in both literature and folk phantasmagoria for many more centuries. as well as some embodied agents - such as the Chakanobi Ningyo tea-serving doll from the Edo Period in Japan (Hornyak 2006), Jacques de Vaucanson's Digesting Duck or Henri Maillardet's and Pierre Jacquet-Droz's anthropomorphic automata from the eighteenth century (Dixon 2004a). As Genevieve Bell puts it:

\section{[I] would say (...) at least three or five thousand years, we have had a fascination with stories about things coming to life (Bell 2018, 26).}

In this sense, we may consider a certain cultural heritage orbiting around the imagination the 'man's alternative' (Telotte 1995), which influences the cultural imagination about robots - what their properties may be, how they can integrate in future society and how they will influence the workforce of the future (McClure 2018). Some scientists and theorists, including Ray Kurzweil, Hans Moravec or Bill Joy, have worked on popularising robotic science, sometimes playing the role of techevangelists who comment on future trends or imagine the future 'aloud' - thus communicating with audiences unaccustomed to the presence of robots.

However, I argue that cultural imagination is also related to the actual robotic research, which in itself covers a diverse range of practices. Even though produced robots can be radically different from fictional ones, the latter still participate in the general phantasmagoria and inspiration or as a paradigm for pursuing further exploration. 
Given that the imaginaries are hardly measurable in quantitative terms and that these domains somewhat overlap, we can take a closer look at the tendencies in the current types of robotic research. These areas influence one another and cross fields to a certain degree, either through a form of joint research between an industry and an artist (such is the case with the robot Kuratas, developed by the artist Kogoro Kurata and built by Suidobashi Heavy Industry) or because many art or DIY projects are simply informed by the technological state-of-the-art and perhaps appropriate existing technology for another purpose. When referring to the scientific research, I predominantly address the institutional scientific experimentation carried out in universities, private or public enterprises and robotic laboratories, bearing in mind that its ultimate aims are primarily instrumental and scientific. When referring to the industrial robotics, I consider a vast range of activities and production for predominantly commercial purposes, from industrial robots to healthcare companions.

I argue that cultural imagination includes both phantasmal and real-life robots, serving as a vast and colourful 'dream-space' and inspiration that motivate roboticists to engage in the actual practices. For example, many Japanese HRI scientists (now in their thirties) grew up watching the Testuwan AtomulAstro Boy series or reading the comics (Kaplan 2004). Artist Kogoro Kurata said that he was inspired by mecha anime when creating Kuratas, especially those in Armoured Trooper Votoms that he watched as a child (Reuters 2012). In an interview for Motherboard, Professor Yoshiyuki Sankai said that he decided to devote his professional development to robotic science after being inspired by Isaac Asimov's book I, Robot (Motherboard 2013). This connection is also mentioned in The Economist's special issue devoted to the technological advancements in robotics:

\footnotetext{
There was very little academic research before there were undergraduates who had seen Star Wars as kids; those former fans have now had a professional lifetime to build academic research groups and spin off companies $(2014,8)$.
}

These areas of research are not always so distinctively separated. Many examples of work fall under several categories, such as Hiroshi Ishiguro's work. Sometimes, a robot is contextualised as an artwork due to its initial non-commercial value and exhibition setting (e.g. SHIRI robot by Nobuhiro Takahashi, Huffpost 2012).

The interdisciplinary character of robotic research has the common goal of pushing the boundaries in technological advancement and reflecting on the different aspects of robots' impact on current and future societies.

\subsection{Robots as techno-dinosaurs}

To illustrate this symbolic connection between the real and the imaginary, I use the dinosaur analogy and briefly introduce W.J.T. Mitchell's (1998) interesting study about dinosaurs as cultural icons. The reason why I use this parable is a certain similarity in cultural status between dinosaurs and robots, which I will explain shortly.

Mitchell stresses the importance of taking into account not only 'the things themselves' (i.e. dinosaurs as the diverse group of reptiles that inhabited the Earth two hundred million years ago) but also their relation to images (representation). Thus, he emphasises the relation between the two groups:

(i) the world of living things (in the case of dinosaurs, an extinct group of animals) and

(ii) the world of images, in which dinosaur images also appear as a particular group or class that is not extinct but in fact, proliferating at a remarkable rate $(1998,58)$.

However, the borderline between natural and artificial is somewhat blurry because a dinosaur, as a long-gone creature, leaves enough room for interpretation, fantasy and speculation. If we apply this analogy to robots, we would notice a fairly similar cultural status. Similar to a dinosaur, a robot is 'a scientific given', whose inner mechanism is only known to a small group of experts. However, its aura as a cultural object reaches out to diverse audiences. Thanks to popular culture (films and TV series in particular) ${ }^{4}$, children and adults worldwide are offered a certain imagetext of robots (Mitchell 1998), which is enveloped in a mystical charisma due to its function as an empty signifier. The core strength of the robot as a cultural icon is not what it is but what it could be, what it can potentially develop into.

Furthermore, in Western popular culture, both figures have a somewhat ambivalent cultural status; they are often represented as ruthless and fearsome monsters - a cultural other (Kim \& Kim 2013) - as well as friendly and anthropomorphic characters (e.g. robot WALL-E (2008) in the animated film with the same name). In some cases, both are represented as a pet or a child-like figure.

The filmic imagery of robots goes one step further, towards their complete disguise as humans (e.g. Ash in Alien [1979], replicants in Blade Runner [1982], etc.). To address this tendency in robotic research and design, Turkle uses the term 'evocative object' of a human (2011, 30). These imageries pose questions, not only about the ontology of the artificial personhood - the intrinsic qualities of a machine that make it humanlike - but also about a human's intrinsic quality that cannot be replaced by a machine. The obvious attempt to cope 
with this challenge is to teach robots to simulate the expression of emotions (see Kismet from the MIT, Wabot from Waseda University and e-Muu from the Eindhoven University of Technology). However, some artists have looked for the answer elsewhere; it is our own bodily vulnerability, our soft tissues that can be manipulated with electric currents, the animal part of us that cannot be simulated by a machine. Thus, the cybernetic phantasmagoria of the body enhanced with technological prosthesis - the figure of a cyborg ${ }^{5}$ - is as prominent in Western cultural imagination as the figure of a robot.

In this regard, we may notice two different streams in both scientific research and robotic art, as follows:

- creation of a robot as an independent agent (as in humanoid robotics or some industrial robots) and

- research on the robotic prostheses and exoskeletons (e.g. Hybrid Assistive Limb by Yoshiyuki Sankai, 2010).

In both cases, robots could be viewed, not only as a technological amalgam for the current and future societies, but also as sources of conflict, anxiety or potential threat (Moravec 1999, 142). These points of doubt have been discussed in robotic art by pushing the limits of the so-called normality in communication, challenging the supposed usefulness of technology for humankind or questioning the body-prosthesis relation.

On the other hand, following Western cybernetic phantasmagoria, we have witnessed some provocative artworks that comment on robotic prosthesis, such as Stelarc's Ping Body (1996) and Laura Beloff's Appendix (2011). Even though they primarily contribute to the discourse about a cyborg, the two artworks showcase a new set of challenges regarding body (and gender) politics. In Stelarc's performance, his bodily movements are caused by an external data system - data is provided by randomly 'pinging' different web domains. The interplay of data is also determined by the web traffic and the distance of a signal, being then 'translated' into electric currents that stimulate his muscles and initiate the movement. Additionally, he wears 'a third arm' - a robotic prosthesis connected to the computer. During his performance, electric stimuli jerk his body against his will, turning it into a 'flesh puppet', a cadaver or, as Dixon notes, 'it makes a direct reference to Deleuze and Guattari's theoretical construct of 'becoming animal' (2004b, 268). Becoming animal is never an imitation; it cannot be acted - we indeed observe a process of mutation, humanity being reduced to animality.

Beloff's piece Appendix offers another relation between the body and technology - it is a networked robotic tail, designed for a human. The person who has the tail attached with cords has no control over its movement. Instead, the tail receives real-time data about the wave height of the sea, as well as real-time update about public transport - these pieces of information are 'translated' into the tail's gesture. With an obvious reference to the phallic surrogate, the robotic tail has a 'will of its own', or rather, falls under the authority of a mystical, faraway centre. Both Stelarc's and Beloff's pieces negotiate the obscurity of the added prosthesis that has a life of its own and indeed represents what Žižek $(2004,173)$ refers to as a 'partial object'6. In these cases, the partial object is the inverted version of a phantom limb (experienced by people who have had their limb amputated). Its obscurity lies precisely in this surplus that subsequently ridicules the 'carrier body'. Stelarc's Extended Arm moves independently from his will, similar to Beloff's Appendix, adding to the uncanniness of these bizarre extensions. Even though the robotic tail does not influence the movement of the body to which it is attached (unlike the hand in Stelarc's performance), in the context of this work, it makes the stark discrepancy between the body and the prosthesis even more uncanny. The hand in the human world and the tail in the animal world are markers of some basic units of body language - carriers of the primordial messages that existed before the system of culture and language. These signifiers are rich with a highly codified system of meanings; however, in both works, they 'talk' noise, chaos or gibberish. Such artworks question the increasing human attachment to technology through a rather literal, organic attachment.

Artworks can thus offer a valuable commentary on current technological trends, often pinpointing their abhorrent or uncanny aspects. In the next section, I discuss this point in detail.

\subsection{What is robotic art?}

Robotic art covers a group of practices that is usually related to the history of computer automated cultural artefacts (CACA) (Penny 2013), although some authors refer to the broader field of mechanically driven kinetic art in an attempt to frame it within an interdisciplinary field of media art practices (Hoetzlein 2009). It spans a range of processes and sensibilities, which Penny (2013) describes as the synthesised skills of the sculptor, installation artist and performer. From a general perspective, a collection of works that includes mechanical agents with a certain level of autonomy is often addressed as robotic art (Kac 1997, Penny 2013), belonging to a broader category of machinic art (Broeckmann 2016), though it can be divided into subcategories, such as 'telerobotic art' (Goldberg 2001, Reichle 2009), 'postbiological life forms' (Reichle 2009), 'robotic installation art', and so on. 
Since the 1960s, information technology-related materials have been introduced to art galleries. The two pioneering exhibitions should be mentioned: Jack Burnham's Software (1970, Jewish Museum, New York, USA) and Jasia Reinhardt's Cybernetic Serendipity (1968, Institute for Contemporary Arts, London). Leaning on the tradition of kinetic art, as well as the phenomenon of an open work ${ }^{7}$ (Eco 1989), one of the main traits in this emerging field involves real-time activities:

\begin{abstract}
Primarily the connection between art and information science was read as a concern with the material spaces of transmission - whether practical, conceptual, social or critical (Ballard 2014, 354).
\end{abstract}

Even today, it seems to be one of the central aspects of robotic art.

It might be argued that robotic art in a broader sense could be understood as encompassing an extensive range of practices that offer commentary about robots and thus are not strictly limited to the production of an actual robot. On the other hand, Penny insists on the sharp division between the two:

Software-based quasi-cinematic practices, (...) which are preoccupied with representation in the naïve sense of 'picturing of something', whilst robotic practices are 'the thing itself' (both the thing itself and representation) $(2013,147)$.

The reader may note here that this view of robotic art is technique oriented and avoids some soft creations borrowed from other media. An example is Geisler's Dream of Beauty 2.0 (1999). In this video piece, the viewer is confronted with a computergenerated image of an attractive female face, which seems to invite the viewer to interact. However, every attempt to establish communication miserably fails, and the only response that the participant eventually receives is a mocking laugh or an airblown kiss from her (Hansen 2006). Ultimately, this piece discusses the codified rules of human communication and how it may be disturbed when we are confronted with the technology that seems to possess the intelligibility we normally expect from another person, but the exchange turns out to be unrewarding. Another already classical work that addresses the problem with human-robot communication is White's Helpless Robot (1987). The robot would ask a visitor for small favours, but after a while, it would become increasingly demanding and even bossy - producing a feeling of discomfort or shame. Both artworks address the ethical and the instrumental aspects of human-robot communication, challenging the perception about robots as always-on-demand artificial actors. Some questions that these artworks pose are as follows: Can we communicate with a robot the same way as with a human? If so, what is the scope of our expectation? Do we approach a robot as an equal, or do we have a secret wish to 'tame it' - make it do what we want? Even though Dream of Beauty 2.0 falls under the category of video art in terms of its technical setting, it certainly sheds some light on the ethical questions about robots and their place in society. Specifically, this artwork puts to the test the symbolic framework of communication that Jerome Bruner (1990) writes about ${ }^{8}$, making the participant aware of the complexity of verbal and non-verbal exchanges in daily life, which is sometimes taken for granted. Bearing this in mind, we can think of technology as the 'mirror' of humankind, its autoreflexive prosthesis, which easily pinpoints what Hansen calls 'nonseamlessness of the interface between embodied human beings and the computer' (2004, 129).

The actual robot's labour, that is, the trace of its activity, has been part of cultural imagination even before the first human-made robot emerged.

One of Henri Maillardet's automata was capable of creating four drawings and writing three poems (The Franklin Institute website). An artificial being who could independently intervene or produce something is one of the central characteristics that contributes to a robot's cultural aura. In fact, Western cultural imagination from late nineteenthand twentieth-century science fiction superimposes superpowers on the machine, and their powers become limitless.

This long-standing fascination with robotic labour, especially robotic creativity, has been explored in artwork, such as Nam June Paik's K-456 robot (1965), introduced as 'the first non-human action artist' (Davis 1973, 67). From more recent examples, I would single out Douglas Bagnall's Film-Making Robot (2004). This robot has developed its own aesthetic sensibility, which Ballard calls 'machine aesthetics' (2014, 354). Bagnall's robot is a technological agent that consists of two parts. Its 'traveling eyes' are part of the device that travels through Wellington on public transport. It uses existing telecommunication networks to download and edit the footage into short clips. At the same time, the rest of the robot's body sits in the gallery, edits the film by assessing each frame, puts together the final version for the day, adds the credits and uploads it on the Internet. In this process, the robot converts every frame into 20 numbers that determine the average brightness of the region of the frame, as well as the red, green, blue (RGB) balance and other technical aspects. Additionally, the robot had some basic 'aesthetic training'; for example, it is programmed to avoid the waypoints that are similar to the recently used ones ('away from recents') and to prefer images at the edge of the space ('away from mean'). It is also trained to like the fine art images found on the web (Bagnall 2011). This artwork shows some interesting aspects. First, the robot's 'art education' does not differ much from 
the approach undertaken by art students becoming introduced to the large amount of visual material, finding meaningful connections and patterns, negotiating visual aesthetics when the primary selection has been made (avoiding repetition and redundancy) and delivering the final product. Second, the robot learns through accumulation of data, analysis, pattern recognition and past experience. Functionally, the same process occurs in the formation of synapses in a newborn; the repetition of a successful attempt (e.g. to catch an object) results in strengthening of a mental synapse, but unsuccessful approaches are gradually abandoned. The idea that a robot can learn and even create, following a learning model similar to that of humans, opens a variety of autoreflexive questions. How is creativity appropriated in the art system today? Can we imagine a future where the credit for an artwork will not bear the name of the artist who has made the robot but only the name of the $\operatorname{robot}^{9}$ ?

\section{CULTURAL IMAGINATION AND DIY PRACTICES}

Thanks to smart mobs (Rheingold 2002), fan networks (Jenkins 2006b), crowdsourcing, intellectual capital spread via social media, open access and 3D printing, phantasmal media has also entered the heterogeneous field of DIY robotic initiatives, making a direct impact on the stream of (currently) non-instrumental/commercial robotics. The 3D printing technology in particular has decreased the cost of the robot-making process, resulting in the proliferation of home-made robots or appropriated versions of commercial robots. As mentioned, these robots are often triggered by the cultural imagination about robots, particularly those that are well branded through dream machines, such as film. Generally, an event such as a robot fair or an exhibition also serves as a kind of 'primordial soup' for later industry implementation, a litmus test for audience acceptance of some new technologies, playing its part in the experience economy (Pine \& Gilmore 1999).

An interesting DIY robotic mob is WALL-E Builders that has a Yahoo! group (now belonging to Oath), which currently counts 2618 members. This group aims to create a WALL-E robot, inspired by the character from Pixar's animated film. One of the members presented his Arduino technology-based robot on a trusted channel on YouTube - a real-life copy of the filmic character that he had worked on for the past five years. Real-life WALL-E is stunningly similar to the movie character and possesses sophisticated motor capabilities that can be controlled via radio. However, the aim of the group is to share the knowledge and the technological solution. Thus, the core product is not the robot itself but a blueprint of its parts and the technology behind it, so it can be used by the other members of the group. Besides this one, several other groups have brought their favourite film characters (e.g. R2D2, T-1000, EVE, Johnny 5, etc.) to artificial life.

This occurrence has a couple of interesting aspects. Similar to every networked system in the beginning of its development, it passes through the premonetary, decentralised model of exchange. Given that the group members are mainly amateur enthusiasts, the structure is horizontal, the data is open, the networked knowledge that is produced is meant to serve the common good. There is another side of the coin, though. The fruits of this free and open exchange can be co-opted by the big players in the industry (including the platform where the exchange takes place) so the aggregate force for common good can all too easily be subverted into aggregate submission for commercial imperatives' (Picone et al. forthcoming). Keeping the exchange open and free becomes an act of political resistance.

Amateur enthusiasts sometimes communicate with the companies that produced the initial artwork. From the company perspective, the work invested in these practices serves as surplus labour, which nevertheless pushes the boundaries of the technological knowledge in the field. Even though many of the produced robots at this stage are basically radio-controlled automata, their fans' pedantic drive to make an exact copy of each robotic character enables them to focus on solving various technical issues. Similar to online discussion groups around fan fiction (Jenkins 2006b), gratification comes from social capital as well as from technological advances.

\section{CONCLUSION}

As information about robotic projects is progressively becoming more available through video channels and social media in general, it inevitably influences the cultural imagination about robots as well. The group of prosumers (Bruns and Scmidt 2011) is growing larger, shifting the approach to different cultural objects. Even if they are not actually engaged in some kind of robot-making activity, prosumers play an active role through the process of sharing and a commenting culture.

Artworks such as Bagnall's Film Making Robot (2004), Robotlab's Manifest (2008), and Obvious' Edmond de Belamy (2017) put the robot at the centre of that process, effectively problematising not only values and hierarchies of artistic ecosystem(s) but also pose some ontological questions: what is creativity? What is an artwork? Who is an artist?

\section{As Šabanović notes:}

While robots as socially intelligent agents are a mirror that reflects our own views of social 
interaction, interacting with these new technologies also changes how we experience and practice sociality $(2010,10)$.

An important way to communicate the narratives revolving around the figure of a robot is through an exhibition targeting a general audience ${ }^{10}$, which provides an opportunity for discussion about recurring discourses in Western media (e.g. the place of robots in the workforce of the future).

As robots progressively become more common in our daily lives, our views on the robot culture will change. Through this process, robotic art will remain an important frontier, a critical terrain that challenges both technological advancements and cultural imagination.

\section{REFERENCES}

Bagnal, D. (2011) A filmmaking robot. Available from: http://halo.gen.nz/robot/ [15.06 2018]

Ballard, S. (2014) Cloud-Watching Robots: Douglas Bagnall's Machine Aesthetics. Convergence: The International Journal of Research into New Media Technologies, 20 (3). 352-368.

Bell, G. (2018) Making life: a brief history of humanrobot interaction, Consumption Markets \& Culture, 21(1). 22-41.

Borggreen, Gunhild. (2011) Cute and Cool in Contemporary Japanese Visual Arts. The Copenhagen Journal of Asian Studies, 29(1). 3960.

Broeckmann. A. (2016) Machine Art in the Twentieth Century. Cambridge, Massachusetts: The MIT Press.

Bruner, J. (1990) Acts of Meaning. Cambridge, Massachusetts: Harvard University Press.

Bruns, A. and Schmidt, J. (2011) Produsage: A Closer Look at Continuing Developments. New Review of Hypermedia and Multimedia. 17(1), 37.

Coeckelbergh, M. (2011) Humans, Animals, and Robots: A Phenomenological Approach to Human-Robot Relations. International Journal of Social Robotics 3(2). 197-204.

Davis, D. (1973) Art and the Future. New York: EP Dutton.

Dixon, S. (2004a) A Brief History of Robots and Automata. The Drama Review, 48(4). 16-25.

Dixon, S. (2004b) Metal Performance: Humanizing Robots, Returning to Nature, and Camping About. The Drama Review. 48(4). 15-46.
Eco, U. (1989) The Open Work. Cambridge, Massachusetts: The MIT Press.

Eyssel, F., de Ruiter, L., Kuchenbrandt, D. (2012) If you sound like me, you must be more human: On the interplay of robot and user features on human robot acceptance and anthropomorphism. In: Proceedings of the 7th ACM/IEEE Conference on Human-Robot Interaction (HRI 2012). Boston, 58 March 2012. Boston: MA.

Fiemnes, S. (2006) The Pervert's Guide to Cinema. (documentary). Manuscript: Slavoj Žižek, Sophie Fiennes. Amoeba Film, Kasander Film Company, Lone Star Productions, Mischief Film.

Geertz, C. (1973) The Interpretation of Cultures. New York: Basic Books.

Goldberg, K. (2000) The Robot in the Garden: Telerobotics and Telepistemology in the Age of the Internet. Cambridge, Massachusetts: The MIT Press.

Hansen, M. (2004) New Philosophy for New Media. Cambridge, Massachusetts: The MIT Press.

Harraway, D. (1991) A Cyborg Manifesto: Science, Technology, and Socialist-Feminism in the Late Twentieth Century. In: Simians, Cyborgs and Women: The Reinvention of Nature. New York: Routledge. 149-181.

Hoetzlein, R. (2009) What is New Media Art? Available from: http://www.rchoetzlein.com/theory/2009/what-isnew-media-art/ [15.06 2018]

Hornyak, T. N. (2006) Loving the Machine: The Art and Science of Japanese Robots. Tokyo: Kodansha International.

Jenkins, H. (2006a) Convergence Culture: Where Old and New Media Collide. New York: New York University Press.

Jenkins, H. (2006b) Fans, Bloggers and Gamers: Exploring Participatory Culture. New York: New York University Press.

Kac, E. (1997) Origin and Development of Robotic Art. Art Journal 56(3). 60-67.

Kakoudaki, D. (2007) Studying Robots, between Science and the Humanities. The International Journal of the Humanities. 5(8). 165-181.

Kaplan, F. (2004) Who is afraid of the humanoid? Investigating cultural differences in the acceptance of robots. International Journal of Humanoid Robotics 1(3). 465-480.

Kavner, L. (2012) Robotic Butt SHIRI Expresses Emotions, Terrifies (VIDEO) Huffington Post. Available from: http://www. huffingtonpost.com/2012/05/17/robotbutt-shiri n 1524229.html [15.06 2018] 
Kim, M. and Kim, E. (2013) Humanoid Robots as "The Cultural Other": are we able to love our creations? Al \& Society, 28(3). 309-318.

Lacan, J. (2006) Écrits. New York, London: W.W. Norton \& Company.

Lövgren, J. and Reimer, B. (2013) Collaborative Media: Production, Consumption and Design Interventions. Cambridge, Massachusetts: The MIT Press.

Lunning, F. (2008) Giant Robots and Superheroes: Manifestations of Divine Power, East and West: An Interview with Crispin Freeman. Mechademia, 3. 274-282.

Magnet, S. (2013) Gender, Sexuality and Engagement in 'Mixed Societies' of Cockroaches and Robots. Women Studies Quarterly. 41(3\&4). 38-55.

McClure, P.K. (2018) "You're Fired," Says the Robot: The Rise of automation in the Workplace, Technophobes, and Fears of Unemployment. Social Science Computer Review. 36(2) 139-156.

Mitchell, W.J.T. (1998) The Last Dinosaur Book: The Life and Times of a Cultural Icon. Chicago: University of Chicago Press.

Moravec, H. (1999) Robot: Mere Machine to Transcendent Mind. Oxford, New York: Oxford University Press.

Mori, M. (1970) The Uncanny Valley. Energy 7(4). 33-35.

Mori, M. (2005) The Buddha in the Robot: A Robot Engineer's Thoughts on Science and Religion. Tokyo: Kosei Publishing.

Motherboard (2013) A Robotic Exoskeleton.
Available https://www.youtube.com/watch?v=25c1hiBKxeE [15.06 2018]

Obvious-art.com (2018). Obvious Art. Available from: http://obvious-art.com/about-us.html [01.09 2018]

Picone, I., Kleut, J. Pavličková, T., Romic, B., MøllerHartley, J., de Ridder, S. (forthcoming) Small acts of engagement. Reconnecting productive audience practices with everyday agency. New Media \& Society (under peer review).

Pine B. J. II and Gilmore J. H. (1998) Welcome to the Experience Economy. Harvard Business Review. July - August. 97-105.

Penny, S. (2013) Art and Robotics: Sixty years of Situated Machines. Al \& Society. 28(2). 146-156.

Reichle, I. (2009) Art in the Age of Technoscience: Genetic Engineering, Robotics, and Artificial Life in Contemporary Art. Vienna, New York: Springer.
Reuters (2012) Japanese man's childhood dreams give birth to giant robot. 28 November. Available from:

http://www.reuters.com/article/2012/11/29/usjapan-giantrobot-idUSBRE8AS04D20121129 [15.06 2018]

Rheingold, H. (2002) Smart Mobs: The Next Social Revolution. Cambridge: Perseus Books Group.

Samani, H., Saadatian, E., Pang, N., Polydorou, D., Owen N., Nakatsu, R. and Koh, J. (2013) Cultural Robotics: The Culture of Robotics and Robotics in Culture. International Journal of Advanced Robotic Systems 10 (400).

Telotte, J.P. (1995) Replications: A Robotic History of the Science Fiction Film. Illinois: University of Illinois.

The Economist (2014) Rise of the Robots: A 14Page Special Report. 29 March - 4 April.

Turkle, S. (2011) Alone Together: Why We Expect More from the Technology and Less from Each Other. New York: Basic Books.

The Franklin Institute. Maillardet's Automaton. Available from: https://www.fi.edu/historyresources/automaton [15.06 2018]

Šabanović, S., Bennett C., and Lee H. R. (2014) Towards Culturally Robust Robots: A Critical Social Perspective on Robotics and Culture. Proceedings of the ACM/IEEE Conference on Human-Robot Interaction (HRI) Workshop on Culture-Aware Robotics (CARS), Germany, 3-6 March. 2014, Bielefeld.

Šabanović, S. 'Emotion in robot cultures: Cultural Models of affect in social robot design' (D\&E2010) (Chicago IL: October 2010).

Vlachos, E., Jochum, E., Schärfe, H. (2016) Head Orientation Behavior of Users and Durations of Playful Open-Ended Interactions with an Android Robot. In: Koh, J., Dunstan, B., Silvera-Tawil, D., Velonaki, M. (eds) Cultural Robotics. LNAI, vol. 9549. Springer: Switzerland

Žižek, S. (2004) Organs Without Bodies: Deleuze and Consequences. New York: Routledge.

\section{ENDNOTES}

1) Here, I use Clifford Geertz's (1966) definition:

[Culture] denotes a historically transmitted pattern of meanings embodied in symbols, a system of inherited conceptions expressed in symbolic forms by means of which [people] communicate, perpetuate, and develop their knowledge about and attitudes toward life (Geertz 1973, 5). 
2) An interesting study on cockroaches shows that the introduction of robots into a closed habitual system influences the behaviour of both robots and insects, resulting in modification of their initial demeanour (Magnet 2013: 42). Insect-like robots were programmed to prefer the lighter shelter, whilst all cockroaches preferred the darker setting. However, after some time spent in cohabitation, a group of cockroaches began to choose the lighter shelter, whilst a group of robots stayed in the darker one. Such studies seem to confirm McLuhan's observation that an introduction of a 'specialist speed-up of exchange and information will serve to fragment the tribal structure' (2008: 26). Even though this experiment was carried out on insects that do not exactly fit under the 'tribal structure' in the human sense, strong cohesive ties that exist in the socially conditioned species indicate this occurrence and should be taken into consideration.

3) The term 'uncanny valley' was coined by Masahiro Mori in 1970 and refers to people's discomfort toward puppets and robots that appear 'human-like' but not quite right.

4) The first film that showed a mechanical dummy capable of interacting with people was Herman C. Raymaker's short silent movie A Clever Dummy (1917). However, robotic characters (or their equivalents in the form of artificial beings) have been present in the literature for a longer period of time even before Karel Čapek's famous play R.U.R. (1927).

5) A cyborg is defined as an organism that combines machinic and organic body parts. In the words of Donna Harraway:

\section{A cyborg is a cybernetic organism, a hybrid of machine and organism, a creature of social reality as well as a creature of fiction (Harraway1991, 149).}

6) The term part (or partial) object is elaborated in the work of Melanie Klein (Lacan 2006, 513), in relation to an infant's underdeveloped perception. Being unable to relate to the whole body, an infant begins relating to his or her mother's breast as a source of immediate gratification; thus, the mother's breast serves as a primordial part-object. The term has also been used in the works of Sigmund Freud, Karl Abraham and Jacques Lacan. However, I refer here to Slavoj Žižek's (Fiennes, 2006) definition, in relation to his concept of organs without bodies; partial objects are related to our bodies but functionally autonomous even though they should not be - producing an eerie feeling or even horror when disembodied. Žižek's example is a scene from David Lynch's film Mulholland Drive (2001); a woman singing on stage suddenly collapses, but her voice goes on (obviously from a pre-recorded tape). Before everyone realises that the voice was prerecorded, there is that split-second horror of a voice divorced from a body and continuing on its own, as a haunting, undead entity.

In his book Organs without Bodies, Žižek explains:

When the [partial] object start[s] to speak, what we hear is the voice of the monstrous, impersonal, empty-machinic subject that does not yet involve subjectivisation (the assumption of an experienced universe of sense) $(2004,173)$.

7) Umberto Eco coined the term open work to describe an artwork that functions as a 'field of possibilities' instead of being an 'end product' (1989, $6)$.

\section{8) Jerome Bruner writes:}

(...) it is culture, not biology, that shapes human life and the human mind, giving meaning to action by situating its underlying intentional states in an interpretive system. It does this by imposing the patterns inherent in the culture's symbolic systems - its language and discourse modes, the forms of logical and narrative explication, and the patterns of mutually dependent communal life $(1990,34)$.

Bruner observes this term from the perspective of the so-called folk psychology, emphasising that 'every social group is organised and held together by some specific psychological tendency or group of tendencies, which give the group a bias in dealings with external circumstances' (Bartlett, in Bruner 1990: 57). A particular culture thus defines its symbolic framework (a horizon of meaning), where normality is defined relationally to the existing amalgam of its symbolic system. By doing so, a specific culture provides space for the formation of constructive memory, which furthermore leads to the formation of stable and persistent institutions and customs. Bruner refers to this constructive memory as 'memory schemata' (58), which influences and determines behavioural (and even ideological) patterns within folk psychology and helps in the quick identification of relationally abnormal or uncanny behaviour.

9) Another recent example is the artwork 'Edmond de Belamy' made by a machine using Generative Adversarial Networks (GAN) technology. The French collective Obvious facilitated the process: 'as the signature of the artist, we wrote the formula of the original GAN model'. (obvious-art.com)

10) One example is the travelling exhibition Hello Robot. (2017-2018) curated by Amelie Klein, Thomas Geisler, Marlies Wirth and Fredo de Smet. Although successful in presenting a comprehensive historical overview of robots in literature, comics, film and art and including many of the key disputes and concerns (e.g. robots and future employment, use of drones in warfare, etc.), the exhibition fails in diminishing the distance between the visitor and a robot. Most of the robots are switched off and 


\section{Robotic Art and Cultural Imagination}

Bojana Romic

displayed in glass cubes. Such a display does not contribute to overcoming the presentation of the robot as the 'other'. 